Обцероссийский математический портал

В. В. Шумилова, Об усреднении задачи с двумя малыми параметрами в среде с двойной пористостью, Матем. заметки, 2003, том 74, выпуск 5, 796-799

DOI: https://doi.org/10.4213/mzm602

Использование Общероссийского математического портала Math-Net.Ru подразумевает, что вы прочитали и согласны с пользовательским соглашением http://www.mathnet.ru/rus/agreement 
Параметры загрузки:

IP : 18.207 .199 .55

26 апреля 2023 г., 17:48:02

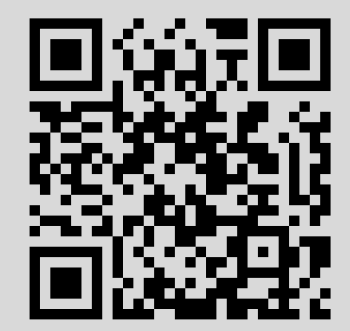




\section{ОБ УСРЕДНЕНИИ ЗАДАЧИ С ДВУМЯ МАЛЫМИ ПАРАМЕТРАМИ В СРЕДЕ С ДВОЙНОЙ ПОРИСТОСТЬЮ}

\section{В.В. Шумилова}

Усреднению задач, описываюших среды с двойной пористостью, посвящено много работ (см. [1] и литературу там). В данной работе для усреднения задачи с двумя малыми параметрами, описывающей среду с двойной пористостью, используется метод обобщенной двухмасштабной сходимости. Отметим, что метод обобщенной двухмасштабной сходимости, введенный В.В. Жиковым [2], применяется к усреднению задач, возникающих при рассмотрении тонких сингулярных структур.

1. Введем необходимые обозначения и определения. Пусть $\mu$ - периодическая борелевская мера на $\mathbb{R}^{N}, \Pi=[0,1)^{N}$ - ячейка периодичности, $\mu(\Pi)=1,\langle\cdot\rangle$ - среднее по мере $\mu$. С мерой $\mu$ свяжем соболевское пространство $H_{\mathrm{per}}^{1}(\Pi, d \mu)$, определяемое как совокупность первых компонент замыкания множества пар $\left\{u, \nabla u: u \in C_{\mathrm{per}}^{\infty}(\Pi)\right\}$ в произведении $L^{2}(\Pi, d \mu) \times L^{2}(\Pi, d \mu)^{N}$. Вторую компоненту этого замыкания условимся называть әрадиентом $u$ и обозначать $\nabla u$. При таком определении градиент функции определен неоднозначно [1]-[3]. Эргодичность меры $\mu$ означает, что функция $u$ есть константа, если она принадлежит $H_{\mathrm{per}}^{1}(\Pi, d \mu)$ и имеет нулевой градиент. Для $a \in L^{2}(\Pi, d \mu), b \in L^{2}(\Pi, d \mu)^{N}$ пишется $a=\operatorname{div} b$ (в смысле меры $\left.\mu\right)$, если $\langle b \cdot \nabla \varphi\rangle=-\langle a \varphi\rangle$, $\varphi \in C_{\text {per }}^{\infty}(\Pi)$. Введем пространство $V_{\text {pot }}(\Pi, d \mu)$ потенциальных векторов как замькание множества $\left\{\nabla \varphi: \varphi \in C_{\text {per }}^{\infty}(\Pi)\right.$ в $L^{2}(\Pi, d \mu)^{N}$. Вектор $b \in L^{2}(\Pi, d \mu)^{N}$ принадлежит пространству $V_{\mathrm{sol}}(\Pi, d \mu)$ соленоидальных векторов, если $\langle b \cdot \nabla \varphi\rangle=0 \forall \varphi \in C_{\mathrm{per}}^{\infty}(\Pi)$.

Пусть $\mu^{h}$ - семейство неотрицательных периодических нормированных мер на $\mathbb{R}^{N}$, слабо сходящихся к мере $\mu, \mu^{h} \rightarrow \mu$ при $h \rightarrow 0$ и $b^{h}$-последовательность функций, ограниченных в пространстве $L^{2}\left(\Pi, d \mu^{h}\right)$.

Работа выполнена при частичной поддержке Российского фонда фундаментальных исследований, грант № 02-01-00114. 


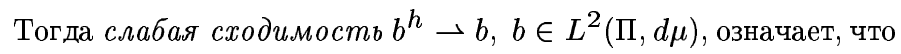

$$
\lim _{h \rightarrow 0} \int_{\Pi} b^{h} \varphi d \mu^{h}=\int_{\Pi} b \varphi d \mu \quad \forall \varphi \in C_{\mathrm{per}}^{\infty}(\Pi) .
$$

Сильная сходимость $b^{h} \rightarrow b, b \in L^{2}(\Pi, d \mu)$, означает, что

$$
\lim _{h \rightarrow 0} \int_{\Pi} b^{h} z^{h} d \mu^{h}=\int_{\Pi} b z d \mu \quad \text { как только } z^{h} \rightarrow z .
$$

Будем предполагать, что выполнены следующие условия:

1) для меры $\mu$ выполнено неравенство Пуанкаре

$$
\int_{\Pi} \varphi^{2} d \mu \leqslant C \int_{\Pi}|\nabla \varphi|^{2} d \mu, \quad \varphi \in C_{\mathrm{per}}^{\infty}(\Pi), \quad\langle\varphi\rangle=0 ;
$$

2) мера $\mu$ невырождена, т.е. не существует ненулевых постоянных потенциальных векторов;

3) выполнены два аппроксимативных свойства, связьвающих меры $\mu$ и $\mu^{h}$ :

(i) для любой функции $a \in C_{\mathrm{per}}^{\infty}(\Pi),\langle a\rangle=0$, найдутся функция $a^{h} \in L^{2}\left(\Pi, d \mu^{h}\right)$ и вектор $b^{h} \in L^{2}\left(\Pi, d \mu^{h}\right)^{N}$ такие, что $\operatorname{div} b^{h}=a^{h}$ (в смысле меры $\left.\mu^{h}\right), a^{h} \rightarrow a$, $b^{h} \rightarrow b$ в $L^{2}\left(\Pi, d \mu^{h}\right)$

(ii) для любого вектора $b \in V_{\text {sol }}(\Pi, d \mu)$ найдется вектор $b^{h} \in V_{\text {sol }}\left(\Pi, d \mu^{h}\right)$ такой, что $b^{h} \rightarrow b$ в $L^{2}\left(\Pi, d \mu^{h}\right)$.

ЗАмеч ание. Аппроксимативные свойства (i), (ii) составляют основное отличие усреднения задач с двумя мальми параметрами от усреднения задач с одним малым параметром [2]. Доказательство этих свойств для различных сингулярных структур можно найти в [2], [4].

2. Введем меру $\mu_{\varepsilon}$ равенством

$$
\mu_{\varepsilon}(B)=\varepsilon^{N} \mu\left(\varepsilon^{-1} B\right)
$$

для любого борелевского множества $B \subset \mathbb{R}^{N}$. Затем при фиксированном $h>0$ определяем скейлинг-меру $\mu_{\varepsilon}^{h}$ по формуле (1). После этого считаем, что $h=h(\varepsilon) \rightarrow 0$. Легко видеть, что $\mu_{\varepsilon}^{h}$ имеет период $\varepsilon$ и слабо сходится к мере Лебега $d x$ при любом $h(\varepsilon) \rightarrow 0$.

Пусть $\Omega$ - ограниченная липшицева область в $\mathbb{R}^{N}$ и $u_{\varepsilon, h}(x)$-последовательность, ограниченная в $L^{2}\left(\Omega, d \mu_{\varepsilon}^{h}\right)$.

ОПРЕДЕЛЕНИЕ 1. Последовательность $u_{\varepsilon, h}(x)$ слабо двухмасштабно сходится к функции $u(x, y) \in L^{2}(\Omega \times \Pi), u_{\varepsilon, h}(x) \stackrel{2}{\rightarrow} u(x, y)$, если для любых $\varphi \in C_{0}^{\infty}(\Omega), b \in C_{\mathrm{per}}^{\infty}(\Pi)$

$$
\lim _{\varepsilon \rightarrow 0} \int_{\Omega} \varphi(x) b\left(\varepsilon^{-1} x\right) u_{\varepsilon, h}(x) d \mu_{\varepsilon}^{h}=\int_{\Omega} \int_{\Pi} \varphi(x) b(y) u(x, y) d x d \mu
$$

ОПРЕДЕЛЕНИЕ 2 . Последовательность $u_{\varepsilon, h}(x)$ сильно двухмасштабно сходится к $u(x, y) \in$ $L^{2}(\Omega \times \Pi), u_{\varepsilon, h}(x) \stackrel{2}{\rightarrow} u(x, y)$, если

$$
\lim _{\varepsilon \rightarrow 0} \int_{\Omega} u_{\varepsilon, h} \nu_{\varepsilon, h}(x) d \mu_{\varepsilon}^{h}=\int_{\Omega} \int_{\Pi} u(x, y) \nu(x, y) d x d \mu,
$$

как только $\nu_{\varepsilon, h}(x) \stackrel{2}{\rightarrow} \nu(x, y)$.

Справедливы следующие теоремы. 
Tеорема 1. Пусть $u_{\varepsilon, h}-$ последовательность из $C_{0}^{\infty}(\Omega)$ такая, что $u_{\varepsilon, h}, \nabla u_{\varepsilon, h}$ ограничень в $L^{2}\left(\Omega, d \mu_{\varepsilon}^{h}\right)$. Тогда (с точностью до вьделения подпоследовательности)

$$
\begin{gathered}
u_{\varepsilon, h}(x) \stackrel{2}{\rightarrow} u(x), \quad u(x) \in H_{0}^{1}(\Omega), \\
\nabla u_{\varepsilon, h}(x) \stackrel{2}{\rightarrow} \nabla u(x)+\nu(x, y), \quad \nu(x, y) \in L^{2}\left(\Omega, V_{\text {pot }}(\Pi, d \mu)\right) .
\end{gathered}
$$

ТЕОРема 2. Пусть $u_{\varepsilon, h}-$ последовательность из $C_{0}^{\infty}(\Omega)$ maкал, что $u_{\varepsilon, h}$, $\varepsilon \nabla u_{\varepsilon, h}(x)$ ограничень в $L^{2}\left(\Pi, d \mu_{\varepsilon}^{h}\right)$. Тогда (с точностью до вьделения подпоследовательности)

$$
\begin{gathered}
u_{\varepsilon, h}(x) \stackrel{2}{\rightarrow} u(x, y), \quad u(x, y) \in L^{2}\left(\Omega, H_{\mathrm{per}}^{1}(\Pi, d \mu)\right), \\
\varepsilon \nabla u_{\varepsilon, h}(x) \stackrel{2}{\rightarrow} \nabla_{y} u(x, y), \quad \nabla_{y} u(x, y) \in L^{2}\left(\Omega, V_{\mathrm{pot}}(\Pi, d \mu)\right) .
\end{gathered}
$$

3. Перейдем к постановке задачи усреднения в некоторых моделях сред с двойной пористостью. Пусть $\mu$ и $\mu^{h}$ - описанные выше меры, удовлетворяющие условиям 1) и 3$), F=F_{1} \cup F_{2} \cup \ldots \cup F_{k}-$ объединение периодических $\mu$-измеримых множеств, непересекающихся в том смысле, что $\exists E_{i} \in$ $H_{\text {per }}^{1}(\Pi, d \mu),\left.\nabla E_{i}\right|_{F}=0,\left.E_{i}\right|_{F_{j}}=\delta_{i j}$ - символ Кронекера; $F^{h}=F_{1}^{h} \cup F_{2}^{h} \cup \cdots \cup F_{k}^{h}$-объединение соответствующих периодических $\mu^{h}$-измеримых множеств.

Кроме того, предполагается, что меры $\left.\mu\right|_{F_{i}}(i=1, \ldots, k)$ эргодичны и невырождены. На мягкую фазу $F_{0}=\mathbb{R}^{N} \backslash F$ никаких условий не накладьвается, кроме $\mu\left(F_{0}\right)>0$.

Обозначим через $\left(F_{i}^{h}\right)^{\varepsilon}=\varepsilon F_{i}^{h}$ гомотетические сжатия множеств $F_{i}^{h}$. Тогда область $\Omega$ распадается на части: $\Omega_{i}^{\varepsilon, h}=\Omega \cap\left(F_{i}^{h}\right)^{\varepsilon}(i=1, \ldots, k)$ - жесткие фазы; $\Omega_{0}^{\varepsilon, h}=\Omega \cap\left(F_{0}^{h}\right)^{\varepsilon}$ - мягкая фаза.

Рассмотрим задачу с двумя малыми параметрами

$$
\begin{gathered}
u_{\varepsilon, h} \in H_{0}^{1}\left(\Omega, d \mu_{\varepsilon}^{h}\right), \quad-\operatorname{div}\left(A_{\varepsilon}^{h} \nabla u_{\varepsilon, h}\right)+u_{\varepsilon, h}=f_{\varepsilon, h}(x), \\
A_{\varepsilon}^{h}(x)= \begin{cases}A_{i}^{h}\left(\varepsilon^{-1} x\right), & \text { если } x \in\left(F_{i}^{h}\right)^{\varepsilon}, i=1, \ldots, k, \\
\varepsilon^{2} A_{0}^{h}\left(\varepsilon^{-1} x\right), & \text { если } x \in\left(F_{0}^{h}\right)^{\varepsilon},\end{cases}
\end{gathered}
$$

где правые части $f_{\varepsilon, h}$ ограничены в $L^{2}\left(\Omega, d \mu_{\varepsilon}^{h}\right), A_{i}^{h}(y)$ - периодические борелевы матрицы $(i=$ $0,1, \ldots, k), A_{i}^{h} \rightarrow A_{i}$ в $L^{2}\left(\Pi, d \mu^{h}\right), A_{i}(y)-\mu$-измеримые матрицы, удовлетворяющие условиям

$$
a_{i}|\xi|^{2} \leqslant A_{i}(y) \xi \cdot \xi \leqslant \alpha_{i}^{-1}|\xi|^{2}, \quad \alpha_{i}>0, \quad \xi \in \mathbb{R}^{N} .
$$

По определению $u_{\varepsilon, h} \in H_{0}^{1}\left(\Omega, d \mu_{\varepsilon}^{h}\right)$ есть решение задачи (2), если

$$
\begin{aligned}
& \sum_{i=1}^{k} \int_{\Omega_{i}^{\varepsilon, h}} A_{i}^{h}\left(\varepsilon^{-1} x\right) \nabla u_{\varepsilon, h} \cdot \nabla \varphi d \mu_{\varepsilon}^{h}+\varepsilon^{2} \int_{\Omega_{0}^{\varepsilon, h}} A_{0}^{h}\left(\varepsilon^{-1} x\right) \nabla u_{\varepsilon, h} \cdot \nabla \varphi d \mu_{\varepsilon}^{h} \\
& \quad+\int_{\Omega} u_{\varepsilon, h} \varphi d \mu_{\varepsilon}^{h}=\int_{\Omega} f_{\varepsilon, h} \varphi d \mu_{\varepsilon}^{h}, \quad \varphi \in C_{0}^{\infty}(\Omega) .
\end{aligned}
$$

Для рассматриваемой задачи опишем предельное уравнение.

Скажем, что функция $u(x, y)$ двух переменных $x \in \Omega$ и $y \in \Pi$ принадлежит классу $V$, если

$$
u \in L^{2}\left(\Omega, H_{\mathrm{per}}^{1}\right) \quad \text { и }\left.\quad \nabla y u\right|_{F}=0,\left.\quad u\right|_{F_{j}}=u_{j}(x) \in H_{0}^{1}(\Omega) .
$$

Заметим, что функцию $u(x, y) \in V$ можно записать в виде [1]:

$$
u(x, y)=\sum_{i=1}^{k} u_{i}(x) E_{i}(y)+u_{0}(x, y), \quad u_{i} \in H_{0}^{1}(\Omega), \quad u_{0} \in L^{2}(\Omega, X),
$$


где $X=\left\{w \in H_{\text {per }}^{1}(\Pi, d \mu):\left.w\right|_{F}=\left.\nabla_{y} w\right|_{F}=0\right\}$.

Пусть $A_{i}^{\text {hom }}$ - усредненные матрицы, соответствующие матрицам $A_{i}$ :

$$
A_{i}^{\mathrm{hom}} \xi \cdot \xi=\inf _{\psi \in C_{\mathrm{per}}^{\infty}(\Pi)} \int_{\Pi \cap F_{i}} A_{i}(\xi+\nabla \psi) \cdot(\xi+\nabla \psi) d \mu, \quad i=1, \ldots, k .
$$

Тогда предельная задача формулируется так: для данного $f(x, y) \in L^{2}(\Omega \times \Pi)$ найти $u \in V$ такую, что выполнено интегральное тождество

$$
\begin{aligned}
& \sum_{i=1}^{k} \int_{\Omega} A_{i}^{\text {hom }} \nabla u_{i} \cdot \nabla \varphi_{i} d x+\int_{\Omega} \int_{\Pi \cap F_{0}} A_{0}(y) \nabla_{y} u \cdot \nabla_{y} \varphi d x d \mu \\
& \quad+\int_{\Omega} \int_{\Pi} u \varphi d x d \mu=\int_{\Omega} \int_{\Pi} f \varphi d x d \mu, \quad \varphi \in V,
\end{aligned}
$$

в котором $\nabla y u$ - некоторый градиент $u$.

Существование и единственность решения задачи (4) как пары $u, \nabla y u$ следуют из теоремы Рисса о представлении линейного функционала в гильбертовом пространстве.

ТЕОРема 3. Пусть правые части $f_{\varepsilon, h}$ в (2) слабо (сильно) двухмасштабно сходятся $\kappa$ $f(x, y) \in L^{2}(\Omega \times \Pi)$. Тогда последовательность решений $u_{\varepsilon, h}(x)$ задач $(2)$ слабо (сильно) двухмасштабно сходится $к$ решению $u(x, y)$ усредненной задачи (4).

ДокАЗАТЕльство. Из тождества (3) следует, что последовательность $u_{\varepsilon, h}$ ограничена в $L^{2}\left(\Omega, d \mu_{\varepsilon}^{h}\right)$. Без ограничения общности считаем, что $u_{\varepsilon, h} \stackrel{2}{\rightarrow} u(x, y)$. Теоремы 1,2 позволяют доказать, что $u(x, y)$ принадлежит классу $V$. Кроме того, из свойств двухмасштабной сходимости [2] следует, что $A_{i}^{h}\left(\varepsilon^{-1} x\right) \nabla u_{\varepsilon, h}(x) \rightarrow A_{i}^{\text {hom }} \nabla u_{i}(x), \varepsilon A_{0}^{h}\left(\varepsilon^{-1} x\right) \nabla u_{\varepsilon, h}(x) \stackrel{2}{\rightarrow} A_{0}(y) \nabla y u(x, y)$.

Теперь в (3) подставим пробную функцию

$$
\varphi(x, y)=\sum_{i=1}^{k} \varphi_{i}(x) E_{i}(y)+\alpha(x) w(y)
$$

где $\alpha, \varphi_{1}, \ldots, \varphi_{k} \in C_{0}^{\infty}(\Omega), w \in X$ и перейдем к пределу при $\varepsilon \rightarrow 0$. В результате получим, что $u(x, y)$ удовлетворяет тождеству (4) на пробных функциях (5). Так как линейная оболочка таких функций плотна в $V[1]$, этого достаточно для того, чтобы $u(x, y)$ было решением предельной задачи.

Нетрудно также получить, что имеет место сильная двухмасштабная сходимость $u_{\varepsilon, h} \stackrel{2}{\rightarrow} u(x, y)$, если только $f_{\varepsilon, h} \stackrel{2}{\rightarrow} f(x, y)$.

\section{СПИСОК ЦИТИРОВАННОЙ ЛИТЕРАТУРЫ}

1. ЖКиков В. В. // Матем. сб. 2000. Т. 191. № 7. С. 31-72. 2. ЖКиков В. В. // Изв. РАН. Сер. матем. 2002. Т. 66. № 2. С. 81-148. 3. ЖКиков В. В. // Матем. сб. 1996. Т. 187. №8. С. 3-40. 4. Chechkin G. A., Jikov V. V., Lukkassen D., Piatnitski A. L. // Asymptotic Anal. 2002. V. 30. №1. P. 61-80. 